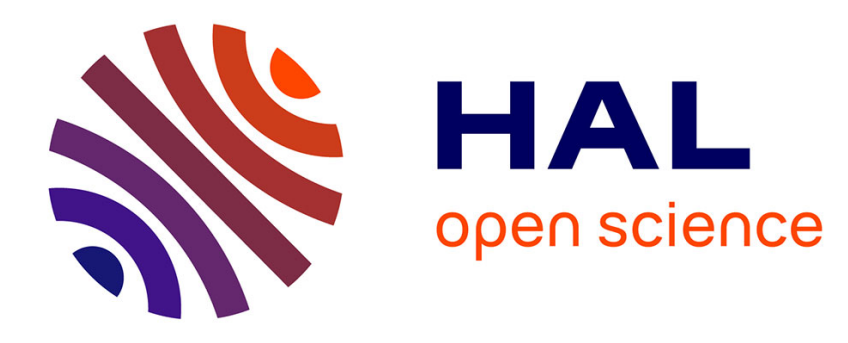

\title{
Search and Localization of a Weak Source with a Multi-Robot Formation
}

Alessandro Renzaglia, Lara Briñon Arranz

\section{To cite this version:}

Alessandro Renzaglia, Lara Briñon Arranz. Search and Localization of a Weak Source with a Multi-Robot Formation. Journal of Intelligent and Robotic Systems, 2020, 97 (3), pp.623-634. 10.1007/s10846-019-01014-0 . hal-02068180

\section{HAL Id: hal-02068180 \\ https://hal.inria.fr/hal-02068180}

Submitted on 15 Mar 2019

HAL is a multi-disciplinary open access archive for the deposit and dissemination of scientific research documents, whether they are published or not. The documents may come from teaching and research institutions in France or abroad, or from public or private research centers.
L'archive ouverte pluridisciplinaire HAL, est destinée au dépôt et à la diffusion de documents scientifiques de niveau recherche, publiés ou non, émanant des établissements d'enseignement et de recherche français ou étrangers, des laboratoires publics ou privés. 


\title{
Search and Localization of a Weak Source with a Multi-Robot Formation
}

\author{
Alessandro Renzaglia and \\ Lara Briñón-Arranz
}

Received: date / Accepted: date

\begin{abstract}
This paper proposes an algorithm to guide a formation of mobile robots, subject to communication constraints, from an arbitrary position to the location of the source of a physical signal in a planar environment. The information on the signal is only based on noisy measurements of its strength collected during the mission and the signal is considered to be weak and indistinguishable from the noise in a large portion of the environment. The goal of the team is thus to search for a reliable signal and finally converge to the source location. An accurate estimation of the signal gradient is obtained by fusing the data gathered by the robots while moving in a circular formation. The algorithm proposed to steer the formation, called Gradient-biased Correlated Random Walk (GCRW), exploits the gradient estimation to bias a correlated random walk, which ensures an efficient non-oriented search motion when far from the source. The resulting strategy is so able to obtain a suitable trade-off between exploration and exploitation. Results obtained in simulated experiments, including comparisons with possible alternatives, are presented to analyze and evaluate the performance of the proposed approach.
\end{abstract}

Keywords Multi-Robot Systems · Source Seeking · Robotic Sensor Networks

\section{Introduction}

Steering a team of autonomous mobile robots over the source of a physical signal is a well studied problem due to its numerous important applications. These can include environmental monitoring [12], search and rescue operations [9], odor source detection [11], pollution sensing [10], etc. In such scenarios, the robots are typically able to sense the environment, collecting and exchanging noisy measurements of the signal strength, and to exploit this information

A. Renzaglia is with Université Grenoble-Alpes, INRIA Grenoble and INSA CITI Lab Lyon, France (email: alessandro.renzaglia@inria.fr). · L. Briñón-Arranz is with Université Grenoble Alpes, CEA, LETI, 38000 Grenoble, France (email: lara.brinon-arranz@cea.fr). 


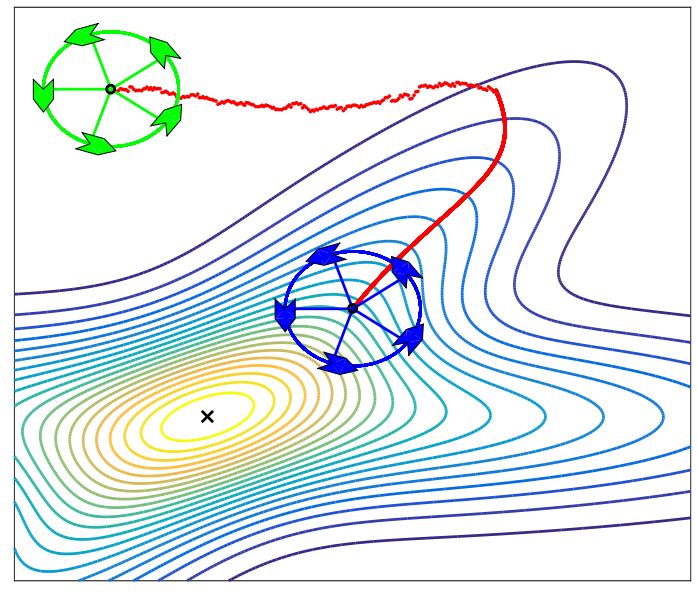

Fig. 1 A multi-robot formation has the goal to reach the location of an emitting source. Initially far from the source (green team), the team firstly needs to explore the environment in search for a signal. When closer (blue team), noisy measurements of the signal can be exploited to estimate the gradient and guide the formation toward the source.

to guide their motion, for instance through an estimation of the signal gradient. However, in most of these applications the signal to locate can be weak or fast decay in space. As a result, in vast portions of the search space the signal-to-noise ratio is such that a reliable estimation of the gradient is impossible to obtain and a non-oriented search strategy becomes essential to ensure success. Moreover, in presence of communication limitations (such as a limited communication range), the robots are not allowed to spread over the environment in an unconstrained way to speed up the search process. Moving in a predefined formation is a well-studied solution [1] for multi-robot teams to overcome this problem and also presents the important advantage to allow exploiting the known geometrical structure of the formation to improve the fusion of collected information. During the task, the formation has, then, to face the classical problem of exploration vs. exploitation, where a right compromise between exploring new areas to improve the current estimation and exploiting the poor available information needs to be found. To solve this problem, deterministic switching strategies between search and gradient descent approaches are too risky since being overconfident about an estimation can mislead the team, while a too conservative approach can be safer but not efficient in terms of convergence time, which is a crucial factor in many real-word applications.

The main contribution of this work is to present a new strategy which instead includes both behaviors, i.e. gradient-based signal exploitation and non-oriented search, and is able to continuously pass from one to another via a probabilistic scheme. The cooperative gradient estimation is achieved by a weighted combination of robots' measurements gathered while they are moving on a circular formation and is based on the results recently presented by one 
of the authors in [5]. This configuration allows the team to obtain an accurate gradient approximation and to estimate the relative error as a function of the formation radius. This approach is embedded in a search strategy based on correlated random walks, ensuring an efficient exploration of the environment in search for an exploitable signal. Finally, it is worth mentioning that the low computational burden of this approach makes it suitable for implementation on very light and constrained robotic platforms.

The rest of the paper is organized as follows. Section 2 provides an overview on work related to non-oriented search and source-seeking problems. The formulation of the specific problem tackled in this paper is then presented in Section 3. Section 4 aims to briefly describe the exploration and gradient estimation approaches which compose our algorithm and Section 5 presents how these strategies are combined to solve the source-seeking problem in the proposed Gradient-biased Correlated Random Walk (GCRW) algorithm. In Section 6 this algorithm is then tested and validated in simulations and results are provided and discussed. Section 7 concludes this paper with final discussions and insights on possible future works.

\section{Related Work}

The problem of using mobile sensors to locate an unknown target (source) while minimizing the search time has been widely studied. In absence of an a priori information on the target location, non-oriented strategies need to be employed to explore the search space. In this scenario, stochastic motions can outperform deterministic strategies and mix local search and global exploration. The most basic of these motions, the simple random walk, presents a diffusive behavior which tends to oversample the search space, and so achieving slow, inefficient exploration. However, more complex processes such as Lévy Walks (LW) and Correlated Random Walks (CRW) have been widely studied in this context, both as optimal search strategies for autonomous agents and to reproduce the movements of several animals in search for prey. A LW is defined by a uniformly random selection of a new orientation and a step length which follows a power-law distribution $p_{\ell}(\ell) \sim \ell^{-\alpha}$, with $1<\alpha \leq 3$. For $\alpha>3$ the movement becomes eventually Gaussian distributed and so the motion is Brownian. Instead, $\alpha \leq 1$ does not corresponds to normalizable distributions. The case of $1<\alpha \leq 2$ is the most interesting where the motion grows ballistically as $\left\langle x^{2}\right\rangle \sim t^{2}$. In particular, Viswanathan et al. prove in [21] that $\alpha \approx 2$ is the optimal value for a search in any dimension when targets are uniformly distributed over the environment. A more comprehensive review of results and insights involving the use of LWs in non-oriented searches can be found in [19]. LWs have been also proposed for multi-robot search problems as in [17], where an artificial potential field is added to create a repulsive force among robots to improve the dispersion process during the search mission.

An alternative motion with similar properties is the CRW, where a persistence in the motion orientation generates a correlation between successive 
positions (CRWs are more extensively presented and rigorously defined in Section 4.1). The relation among these two stochastic processes has been analyzed in [15] and in [2] a study on the search efficiency in terms of motion correlation is presented.

The literature on source seeking problems with mobile robots has rapidly grown in recent years. Interesting and extensive reviews on source localization approaches for environmental monitoring can be found in [7] and, for more recent developments, in [3]. A vast family of algorithms is based on the idea of Chemotaxis, where a single or multiple robots follow the - estimated - local gradient to locate the source. In [6], Chang et al. propose a multi-agent approach to transform the detected turbulent plume field into a smoother scalar field, conserving the same source but with a better defined gradient. All the Chemotaxis-based approaches rely on the presence of a well-defined gradient in the signal field and cannot manage a signal completely covered by noise. A study of reactive single-robot algorithms which deal also with the search part is presented in [16] for the problem of plume detection, where information is still present but only in an intermittent and sparse way. Considering the same scenario, i.e. where patches of detectable odors or chemical components are diffused over the environment by turbulent flows, a gradient-free method called Infotaxis was presented in [18]. In this algorithm, the search for the source is guided by a maximization of the expected rate of information gain defined through Shannon's entropy. In [8], an extension of this approach for multirobot systems has been recently proposed. Pasternak et al. presented in [13] a different approach for the same problem but based on LWs to exploit the cues detected in the flow. These strategies, known as Lévytaxis, share with ours the use of similar stochastic motions to guide the searcher but are strongly based on the presence of a current or air flow which could guide the searcher toward the source. In [13] for example, no measure of a signal strength is used for the strategy, which is, instead, based only on the flow structure. In our problem, we do not assume the presence of any detectable current in the environment.

This paper aims to deal simultaneously with the two aforementioned problems, proposing a new probabilistic scheme to mix non-oriented optimal search with gradient-based source localization for a team of mobile robots. This global strategy significantly extends the contribution presented in [5], where only the gradient-based part is presented and no possibility to deal with highly noisy scenarios was present. Note that, although many optimization algorithms adopt a global search strategy to find a coarse solution and/or escape local optima mixed with a local search to refine the solution and possibly converge to the global optima, these solutions are not trivially adaptable to the considered problem. In fact, even though the two domains are strictly related, in most of the optimization techniques, the way to include global properties does not take into account the constraints of a physical system called to explore a real environment, such as: cost of traveling, gathering measurements, smoothness of trajectory, etc, contrary to our case. For these reasons, we believe the novelty in the contribution of this paper can be identified not only with respect 
to the previous publication [5] but, more largely, to the current literature on the subject.

\section{Problem Formulation}

The objective of this work is to design a strategy to steer a team of cooperating robots with limited communication capabilities to the location of a signal source in a planar bounded environment. The robots do not have any prior knowledge on the source location and acquire information on-line through noisy measurements of the signal strength.

\subsection{Robots' Formation}

In order to enhance the robots capability to rightfully estimate the signal gradient as a team, we exploit their collaboration by deploying them in uniformly distributed patterns along a circular formation. We consider here such a configuration for two main reasons. Firstly, as discussed more thoroughly in Section 4.2, fusing measurements collected by a group of sensors uniformly distributed in a circle allows obtaining an accurate estimation of the gradient at the center of the circle. Secondly, many commonly used underwater and aerial robots are not able to stop at a given position (the source location in our case) due to their physical constraints, for example if their linear velocity cannot be reduced to zero. In these cases, a circular motion is a perfect way to encircle the source when located, respecting the system constraints.

Let us consider a group of $N$ robots stabilized to a planar uniform distributed circular formation described by a radius $R$, a rotation angle $\varphi_{t}^{0}=\omega_{0} t$ where $\omega_{0}$ is a constant rotation velocity, and a given center point $\mathbf{c} \in \mathbb{R}^{2}$. The position of robot $i$ at instant $t$ is given by the following equation:

$$
\mathbf{r}_{t}^{i}=\mathbf{c}_{t}+R\left[\begin{array}{c}
\cos \varphi_{t}^{i} \\
\sin \varphi_{t}^{i}
\end{array}\right], \quad i=1, \ldots, N
$$

where

$$
\varphi_{t}^{i}=\varphi_{t}^{0}+i \frac{2 \pi}{N}
$$

is the rotation angle.

There are several works dealing with the control of circular formations for mobile robots. If the robots represent non-holonomic vehicles modeled by unicycle-like dynamics, the cooperative circular control law presented in the previous work [4] ensures the convergence of a team of robots to a circular formation whose center is time-varying. Inspired by the synchronization problem of coupled oscillators, the introduction of a potential function in the control law makes the robots converge to an evenly spaced configuration, i.e. with the rotation angle $\varphi_{t}^{i}$ satisfying eq. (2) for $i=1, \ldots, N$. In order to achieve this uniform distribution along the circle, the cooperative control law relies only 
on local information, specifically on the relative heading angles between each robot and its neighbors. This local information can be directly measured by the robots or transmitted via a communication network.

For the sake of clarity, we present here the main ideas of the time-varying circular formation control proposed in [4]. In the proposed approach, the key idea is to generate circular trajectories with a properly chosen, stable virtual system and to enforce the robots to track them. The autonomous virtual system is modeled by unicycle dynamics with constant linear velocity as follows:

$$
\begin{aligned}
\dot{\hat{\mathbf{r}}}_{t}^{i} & =\left|\omega_{0}\right| R\left[\begin{array}{c}
\cos \psi_{t}^{i} \\
\sin \psi_{t}^{i}
\end{array}\right], \\
\dot{\psi}_{t}^{i} & =u_{t}^{i},
\end{aligned}
$$

where $\hat{\mathbf{r}}_{t}^{i}$ is the position vector of the virtual agent $i$ at time $t, \psi_{t}^{i}$ its heading angle and $u_{t}^{i}$ the control input. Communication between agents is considered in order to achieve a uniform distribution around the desired circular formation. In the set-up adopted in this paper, each robot communicates the virtual quantity $\psi^{i}$ to its neighbors through an undirected communication graph. A limited range communication can be considered in this case, since the robots only need to communicate with their two nearest neighbors. Each virtual agent is stabilized to a fixed circular motion with radius $R$ and angular velocity $\omega_{0}$ around the origin thanks to the following circular control:

$$
\begin{aligned}
u_{t}^{i} & =\omega_{0}\left(1+\kappa \hat{\mathbf{r}}_{t}^{i T} \hat{\mathbf{r}}_{t}^{i}\right)-\frac{\partial U}{\partial \psi^{i}}, \\
\frac{\partial U}{\partial \psi_{t}^{i}} & =\frac{\kappa_{u}}{\left|\mathcal{N}_{i}\right|} \sum_{j \in \mathcal{N}_{i}} \sin \left(\psi_{t}^{i}-\psi_{t}^{j}\right),
\end{aligned}
$$

where $\kappa>0$ and $\kappa_{u}>0$ are two control parameters, $\mathcal{N}_{i}$ is the set of neighbors of agent $i$ and $\left|\mathcal{N}_{i}\right|$ denote its number of neighbors. Using Lyapunov techniques and thanks to the LaSalle's Invariance Principle, it can be proven that the virtual system dynamics converge asymptotically to:

$$
\dot{\hat{\mathbf{r}}}_{t}^{i}=\omega_{0} R \hat{\mathbf{r}}_{t}^{i}
$$

which corresponds to a circular motion around the origin. Additionally, the gradient term enforces each virtual agent to move away from its neighbors until the equilibrium point, corresponding to the evenly spaced configuration in the case of connected graphs, is reached (details of the proof can be found in [4]). The circular trajectories of the autonomous virtual system $\hat{\mathbf{r}}^{i}$ are then considered as references to be tracked by the relative vectors $\mathbf{r}_{t}^{i}-\mathbf{c}_{t}$. A simple tracking controller can be designed to enforce robots' dynamics to converge to the desired trajectory $\hat{\mathbf{r}}_{t}^{i}+\mathbf{c}_{t}$ and consequently to stabilize the robots to an evenly spaced circular motion around the time-varying center $\mathbf{c}_{t}$, as described in (1). 


\subsection{Signal Strength}

Each robot represents a mobile sensor or a vehicle equipped with a sensor that is able to measure the signal strength emitted by the source. In mathematical terms, the signal distribution emitted by the source is a bi-dimensional spatial function representing the scalar field with a maximum (or minimum, depending on the nature of the signal) in the position where the source is located. The distribution of the signal strength in the environment is described by an unknown positive spatial mapping $\sigma(\mathbf{r}): \mathbb{R}^{2} \rightarrow \mathbb{R}^{+}$, so that robot $i$ measures the signal strength at time $t$ at its position $\mathbf{r}_{t}^{i}$ as $\sigma\left(\mathbf{r}_{t}^{i}\right)$. We do not consider here the case of multiple emitting sources in the environment, i.e. the source located at $\mathbf{r}^{*}$ is the only maximum of the scalar field.

In this work, we focus on scenarios where the signal strength and its decay in space compared with the dimension of the search area and noise in measurements is such that it can be considered zero and/or indistinguishable from the noise in a large part of the environment. The model of the signal is considered to be unknown and the robots are only able to measure the signal strength at their current locations.

Let $\nabla \sigma(\mathbf{r}) \in \mathbb{R}^{2}$ denote the gradient vector at $\mathbf{r}$ and $\mathbf{H}_{\sigma(\mathbf{r})}$ the corresponding Hessian matrix. We assume that the signal strength is smooth enough to consider that the Hessian matrix is bounded. This assumption allows a large class of functions to represent the signal strength of the scalar field of interest. For instance, several physical quantities, as electrical and magnetic fields, light, radiation and sound follow inverse-square laws. Therefore, in these cases, the intensity of linear waves radiating from a point source is inversely proportional to the square of the distance from the source, satisfying the smoothness assumption. Diffusion processes as temperature and chemical concentration also satisfy this assumption.

\section{Source Localization}

The studied problem could be ideally separated in two different sub-tasks: the signal search phase and the subsequent source estimation. In this scenario the robots should switch strategy when the first objective is achieved. However, the presence of noise makes the knowledge on the signal strength often unreliable and employing a single strategy able to optimally carry on both phases and continuously pass from one to the other can be a more suitable and robust strategy. In particular, such a solution can guide the team during the transition between the two phases, where a strong signal is not yet available but weak noisy measurements can still help to bias the search strategy improving the results.

The proposed approach is based on CRWs biased by the estimation of the signal gradient. Let us first describe the two different strategies and in Section 5 we present the proposed algorithm which combines both of them via a probabilistic scheme without the necessity of discontinuous transition. 

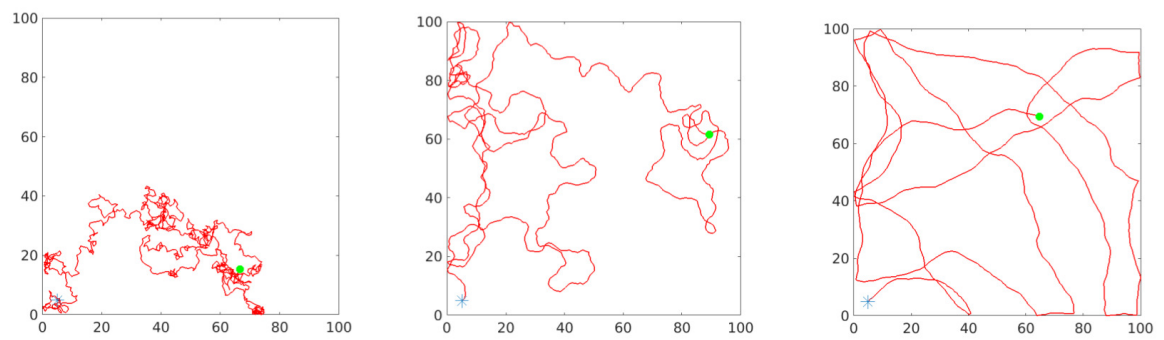

Fig. 2 CRWs in a bounded environment with a different value of correlation: left $k=1$, middle $k=5$ and right $k=50$. The blue stars indicate the starting points, the green points the ending ones.

\subsection{Exploration: Correlated Random Walk}

A CRW is a stochastic motion characterized by a short-term correlation in the direction of movement. Such a correlation between successive step orientations, also known as persistence, produces a local biases and the walker is more likely to maintain a similar direction to its previous one. This fundamental statistical property is mathematically described by a unimodal probability distribution of angles centered around the previous direction. Angular distributions, defined on periodic intervals, are inherently different from classic distributions and are usually constructed by wrapping the usual definition on the real line, i.e.:

$$
f_{\text {wrap }}(\theta)=\sum_{k=-\infty}^{+\infty} f(\theta+2 \pi k), \quad \theta \in[-\pi, \pi) \text {. }
$$

However, closed form expressions following this definition are possible in very few cases. In this paper, we adopt the well known von Mises distribution, which is a close version of the wrapped normal distribution:

$$
\Phi(\theta)=\frac{1}{2 \pi I_{0}(k)} e^{-k(\theta-\mu)}
$$

where $I_{0}(\cdot)$ is the zero-th order modified Bessel function of the first kind, $k$ regulates the process correlation $(1 / k$ is the analogous of the variance in the normal distribution) and the mean is, in our case, the previous step orientation $\mu=\theta_{t-1}$.

In terms of movement steps, as for the simple random walk, their length is constant at every time, and hereafter considered unitary without any loss of generality. It is worth mentioning the difference with respect to the aforementioned Lévy walks, where steps have a varying length, following a determined probability distribution, and the directions are uniformly distributed in $[0,2 \pi)$. Additionally, if an external bias is also present, influencing the determination of the new direction, the resulting process is usually known as Biased Correlated Random Walk (BCRW) [14]. 
The presence of the motion inertia has crucial effects on the exploration properties of CRWs. In fact, for limited time scales, shorter than a certain correlation time $\tau$, CRWs can be considered super-diffusive processes, and so significantly outperforming standard Brownian motions in terms of covering capabilities. Then, at sufficiently larger times a normal diffusion behavior always emerges [20]. This phenomenon can be proved by considering the Mean Square Displacement MSD $=\left\langle\left(x-x_{0}\right)^{2}\right\rangle$, which can be considered a measure of the portion of explored space by a stochastic motion. For long enough time $t$, RWs scale as $t^{\nu}$, where $\nu=1$ in the standard Brownian motions. On the other hand, in CRWs $\nu>1$ for $t<\tau$ (and in particular $\nu=2$ for continuous time CRWs) [2]. An explicit expression for $\tau$ as a function of the correlation is given by $\tau \simeq-1 / \ln (\langle\cos (\theta)\rangle)$, where $\langle\cos (\theta)\rangle=I_{1}(k) / I_{0}(k)$ for the von-Mises distribution [2].

Fig. 2 shows an example of CRWs corresponding to increasing value of correlation $k$, namely for $k=1,5,50$. It is clear how a higher persistence in the orientation reduces the path tortuosity and controls the trade-off between local and global exploration.

\subsection{Exploitation: Gradient Estimation}

Besides to a non-oriented exploration strategy to search for a signal, to efficiently solve our task we also need a reliable gradient estimation technique to exploit the information when available. To tackle this problem we rely on the results presented in [5]. In presence of noisy signal measurements, a circular formation has been proven to be a suitable configuration to cooperatively estimate the gradient vector. This estimation is then used to guide the center of the formation towards the source allowing the team to deploy around it and estimate its location with precision. During its motion, each robot measures the signal strength at its current location and transmits this information to the rest of the fleet in order to cooperatively estimate the gradient. The following lemma formalizes the result on cooperative estimation of the signal gradient at the center of a circular formation:

Lemma 1 Let $\sigma: \mathbb{R}^{2} \rightarrow \mathbb{R}^{+}$be a bounded function and $\sigma\left(\boldsymbol{r}^{i}\right)$ be the measure collected by robot $i$, where $\boldsymbol{r}^{i}$ is its position vector given by (1). Considering a fleet of $N \geq 3$ robots uniformly distributed along a circular formation centered at $\boldsymbol{c}$ with radius $R$, the following equation for the estimated gradient $\hat{\nabla} \sigma(\boldsymbol{c})$ holds:

$$
\hat{\nabla} \sigma(\boldsymbol{c})=\frac{2}{R^{2} N} \sum_{i=1}^{N} \sigma\left(\boldsymbol{r}^{i}\right)\left(\boldsymbol{r}^{i}-\boldsymbol{c}\right)=\nabla \sigma(\boldsymbol{c})+\boldsymbol{\Psi}(R, \boldsymbol{c}),
$$

where the approximation error term $\boldsymbol{\Psi}(R, \boldsymbol{c})$ satisfies

$$
\|\boldsymbol{\Psi}(R, \boldsymbol{c})\| \leq \lambda_{\max }\left(\boldsymbol{H}_{\sigma(\mathbf{r})}\right) R,
$$

with $\lambda_{\max }$ representing the largest eigenvalue of the Hessian matrix. 
The details of the proof, based on Taylor series expansion, can be found in [5]. It is important to highlight that this result holds only for circular formations. The symmetric properties of an evenly spaced circular formation of robots, in particular the fact that the robots' positions satisfy $\sum_{i=1}^{N} \mathbf{r}^{i}-\mathbf{c}=0$, are crucial to prove the previous lemma. Note that in this previous work it is also proven that for quadratic signals, which represent a good approximation of the true signal near the source, the computation of the gradient is exact, i.e., eq. (8) is satisfied with $\boldsymbol{\Psi}(R, \mathbf{c})=0$.

As a result, as proven by Lemma 1, the interest of using a circular formation is to exploit its symmetric properties in a way that the gradient estimation can be obtained via simple averages that involve only the products of the measurements collected by the robots and their relative position with respect to the formation center. Note that the smoothness assumption on the signal, i.e., that the Hessian matrix is bounded, is only required in order to obtain a bound on the approximation error.

We consider that the signal measurements collected by the robots are corrupted by white zero-mean Gaussian noise, i.e., $\sigma\left(\mathbf{r}^{i}\right)+\omega^{i}$ where $\omega^{i} \sim N\left(0, \xi_{\omega}^{2}\right)$. Both the expectation and the variance of the additional estimation error due to the noise are studied in [5]. It has been proven that the expectation is not affected by Gaussian noise, i.e.

$$
\mathbb{E}\left[\frac{2}{R^{2} N} \sum_{i=1}^{N}\left(\sigma\left(\mathbf{r}^{i}\right)+\omega^{i}\right)\left(\mathbf{r}^{i}-\mathbf{c}\right)\right]=\hat{\nabla} \sigma(\mathbf{c})
$$

and the variance of the error induced by the noisy measurements is inversely proportional to the radius squared, i.e.

$$
\operatorname{Var}\left[\frac{2}{R^{2} N} \sum_{i=1}^{N} \omega^{i}\left(\mathbf{r}^{i}-\mathbf{c}\right)\right]=\frac{2}{R^{2} N} \xi_{\omega} .
$$

Therefore, the greater the radius value, the smaller the influence of noise in the gradient estimation. However, as proven in Lemma 1, the error term $\mathbf{\Psi}(R, \mathbf{c})$ vanishes when the radius tends to zero. Consequently, we conclude that the radius has an important role in the precision of the gradient estimation and for the attenuation of the noise effects.

The signal gradient estimated by the circular formation of robots provides useful information to be exploited by our proposed searching strategy. The direction and norm of the estimated gradient can be applied as external bias of a CRW-based algorithm. Based on previously presented results on gradient estimation, we are now able to compute the direction of the estimated gradient $\hat{\nabla} \sigma(\mathbf{c})$ at each time step as

$$
\theta_{t}^{G}=\arctan \left(\mathbf{e}_{2}^{T} \hat{\nabla} \sigma(\mathbf{c}) / \mathbf{e}_{1}^{T} \hat{\nabla} \sigma(\mathbf{c})\right),
$$

where $\mathbf{e}_{1}=[1,0]^{T}$ and $\mathbf{e}_{2}=[0,1]^{T}$ are basis vectors of the global inertial coordinate frame. 


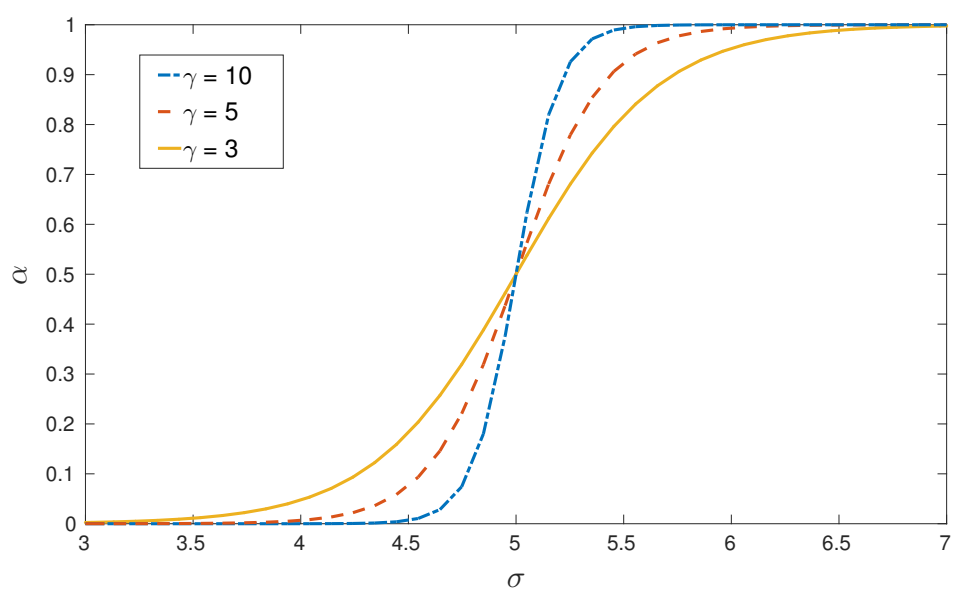

Fig. 3 Activation function in $\alpha$ with $\sigma_{0}=5$ and $\gamma=10,5,3$.

\section{GCRW Algorithm}

The main idea behind the proposed algorithm is to design a probabilistic scheme, which allows a continuous transition between the two previously presented approaches for exploration and exploitation of the detected signal, to steer the center of the circular formation. This transition needs to be regulated by the signal-to-noise ratio, which represents an indication of how reliable is the collected information. To quantify this concept of reliability, we introduce the following function $\alpha_{t}$ :

$$
\alpha_{t}(\bar{\sigma})=\frac{1}{1+e^{-\gamma\left(\bar{\sigma}_{t}-\sigma_{0}\right)}} \cos ^{2}\left(\frac{\theta_{t}^{G}-\theta_{t-1}^{G}}{2}\right),
$$

where $\bar{\sigma}$ is the mean signal measured by the robots, i.e.

$$
\bar{\sigma}=\frac{1}{N} \sum_{i=1}^{N} \sigma\left(\mathbf{r}^{i}\right) .
$$

The first term in $\alpha$ is a classic choice for an activation function, where $\gamma$ and $\sigma_{0}$ are user-defined parameters defining the sigmoid's steepness and midpoint respectively (see Fig. 3). The second term tends to trust more gradient estimations which are consistent in two consecutive iterations. In other words, is maximum where the two estimated gradients are collinear and tends to zero when the angular difference tends to $\pi$.

The function $\alpha$ is then used to construct a new probability distribution for the random walk orientation, where the estimation of the gradient is combined to the von Mises distribution, playing the role of a bias. The final probability distribution is so defined as:

$$
P_{t}(\theta)=\left(1-\alpha_{t}\right) \Phi_{t}(\theta)+\alpha_{t} \delta\left(\theta-\theta_{t}^{G}\right),
$$


where $\Phi(\cdot)$ is the von Mises distribution defined in eq. $(7), \delta(\cdot)$ is the Dirac delta distribution and $\theta_{t}^{G}$ is the estimated gradient direction defined in eq. (11). In the limit cases of a very weak or very strong signal, the effects from the gradient estimation and from the random motion, respectively, become irrelevant. However, in the more interesting intermediate zone, the probabilistic selection of the strategy weighted by the function $\alpha$ produces a smooth behavior and a more robust response to random fluctuations generated by the noisy sensors.

In an analogous way, the distance traveled by the formation center at each iteration is given by:

$$
\Delta_{t}=\left(1-\alpha_{t}\right)+\alpha_{t} \nu\|\hat{\nabla} \sigma(\mathbf{c})\|,
$$

where $\nu$ is the classic step size of the gradient descent algorithm and $\|\cdot\|$ the Euclidean norm. Additionally, a maximum speed constraint is considered, i.e. $\Delta_{t} \leq \Delta_{\max }$. The expression in (15) ensures a unitary movement in the exploration phase and a step depending on the gradient in the opposite limit case to guarantee convergence onto the source.

\section{Simulation Results}

In this section, we test the GCRW algorithm in simulations where the robots' formation is called to locate a signal source in a bounded environment. Different signal models are considered to show the efficiency of the proposed approach. Particular attention is given to the study of the effect of the correlation in the random walk process and the robustness of the algorithm with respect to changes in its parameters. The performance is evaluated in terms of success rate in locating the source given a limited time budget and average time to complete the mission. In all simulations, the robots move in a bounded environment, with a maximum speed of $1 \mathrm{~m} / \mathrm{s}$, where a source of unknown position emits a signal whose strength prevails over the noise only in a limited region. The team is composed of six robots moving in a circular formation of radius $R=5 m^{1}$. The noise on the measurements is modeled as a zero-mean Gaussian noise, where the variance will be specified in each case.

\subsection{Illustrative examples}

Fig. 4 presents a first illustrative example showing the importance of including an exploration strategy to the gradient-based source seeking approach in such a scenario. In this case, the scalar field representing the signal, presenting non-convex level curves and having its source in $(0,0)$, is given by:

$$
\sigma(\mathbf{r})=a\left(\exp \left(-\mathbf{r}^{T} S_{1} \mathbf{r}\right)+\exp \left(-\mathbf{r}^{T} \Theta_{\frac{\pi}{4}}^{T} S_{2} \Theta_{\frac{\pi}{4}} \mathbf{r}\right)\right),
$$

${ }^{1}$ For an exhaustive analysis of the source seeking algorithm varying the number of robots and formation radius see [5]. 

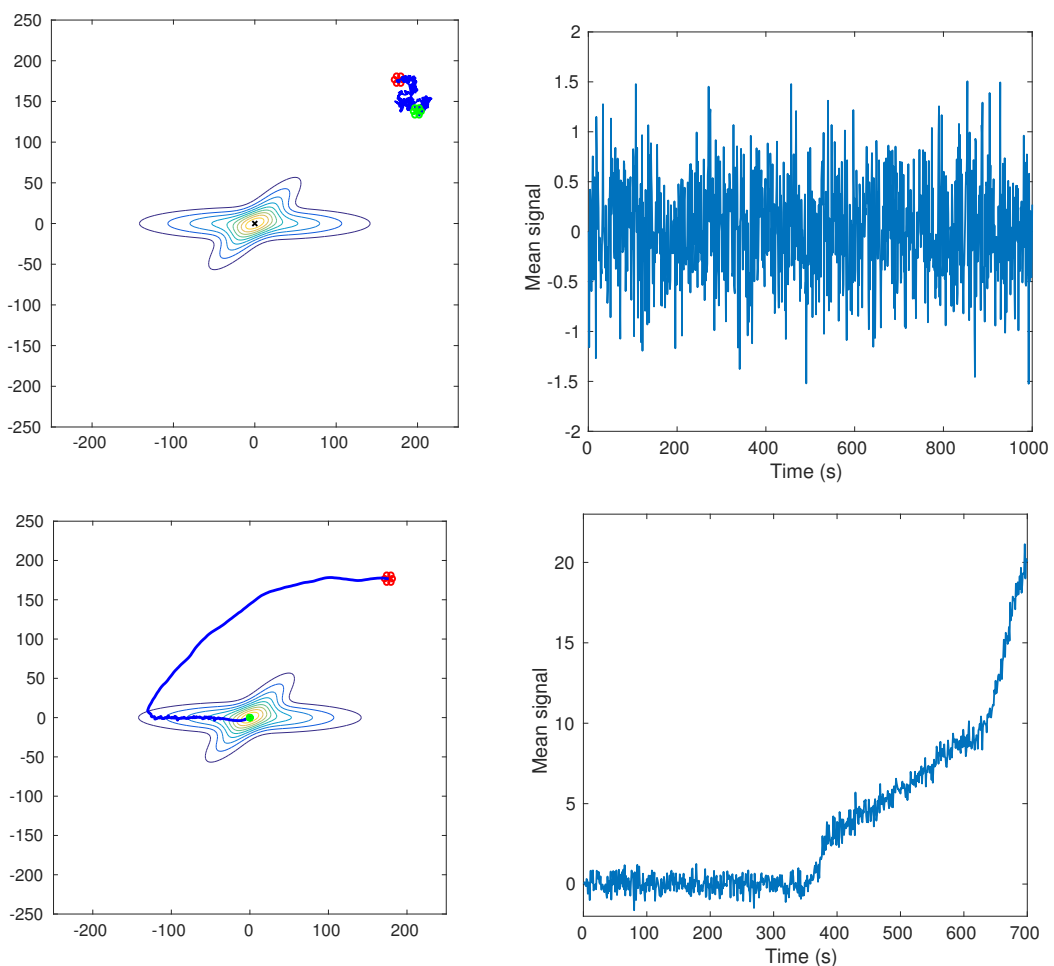

Fig. 4 The robots move in a bounded square region of side $250 \mathrm{~m}$ with a maximum speed of $1 \mathrm{~m} / \mathrm{s}$. Top: at the team initial position the noise prevails over the signal and with only the gradient estimation the robots fail to find the source. Bottom: The GCRW algorithm allows the team to start exploring the environment until a reliable signal lead to correctly locate the source.

where

$$
a=10, S_{1}=\frac{1}{250}\left[\begin{array}{cc}
\frac{1}{\sqrt{50}} & 0 \\
0 & 1
\end{array}\right], S_{2}=\frac{1}{250}\left[\begin{array}{ll}
1 & 0 \\
0 & \frac{1}{\sqrt{15}}
\end{array}\right]
$$

and $\Theta_{(\cdot)}$ representing the rotation matrix. At the starting position the signal is too weak compared to the noise (here having a standard deviation $\xi=0.5$ ) and the team is not able to obtain a consistent gradient estimation to guide its search. The resulting trajectory is thus only a random movement close to the initial position and the robots fail to locate the source. On the other side, by adopting the GCRW algorithm, the initial signal strength is such that the exploration component initially prevails and the team is finally able to find the signal and progressively be led by a reliable gradient estimation, converging onto the source.

Fig. 5 presents a scenario with a more complex signal field, including multiple sources (maxima). As expected, the gradient-based nature of the algorithm assures the convergence to a local maximum and the result is so depending on the initial configuration. However, it is worth noting that the stochastic 

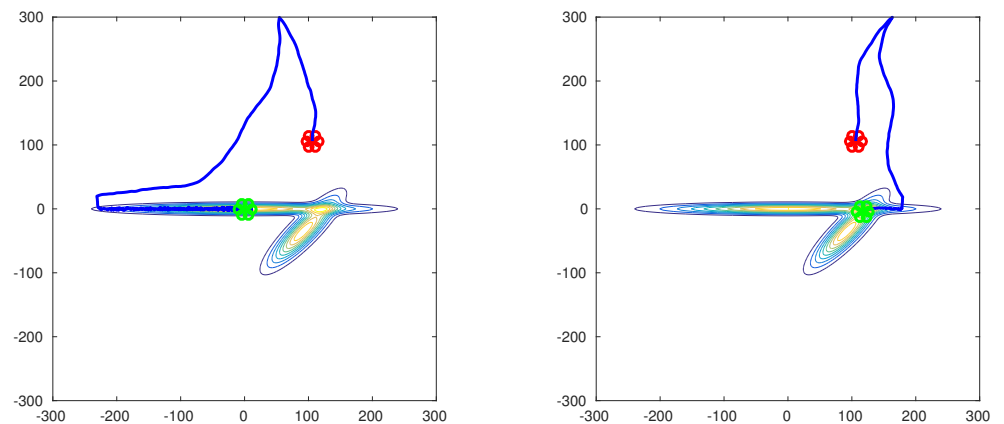

Fig. 5 More complex signal presenting multiple sources. Due to the stochastic nature of the exploration phase, the team can converge to a different source even starting from the same initial conditions.

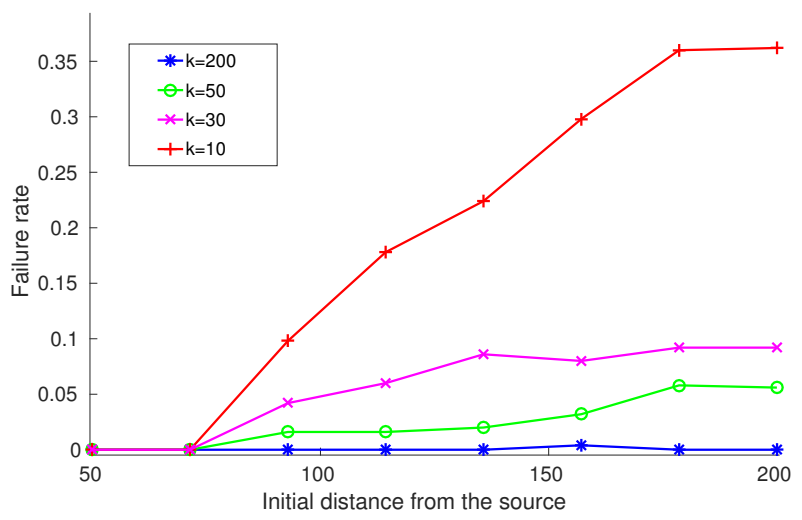

Fig. 6 Rate of failure in locating the source given a maximum mission time of $3000 \mathrm{~s}$ as a function of the initial distance from the source for four different values of the random walk correlation $k$.

exploration of the non-informative region can lead the formation to converge to different maxima even when starting from the same initial conditions.

\subsection{Algorithm Evaluation}

To give more quantitative information on the importance of having correlation in the random walking process to deal with the search phase, we carried out the following test. Given a maximum time budget to achieve the mission, we compare the rate of failures in encountering the source as a function of the initial distance of the formation center from the source for different values of the parameter $k$, which regulates the correlation as shown in Fig. 2. Considering 
a maximum mission time is fundamental in many real applications where the limited energy budget of the robots can represent a hard constraint to deal with and/or a fast localization of the source can be crucial. The results are presented in Fig. 6 and correspond to the average obtained over 500 trials, for a maximum time budget of $3000 \mathrm{~s}$. The parameters in $\alpha(\bar{\sigma})$ are fixed as $\sigma_{0}=3$ and $\gamma=10$. From this result, it is clear that, starting from a distance greater than $75 \mathrm{~m}$ the exploration becomes crucial and low values of correlations lead to a poor covering capability and, consequently, search efficiency.

A second, and even more important aspect to investigate is the effect of the probabilistic scheme employed to combine the exploration and exploitation phases. To do so, we compare our approach with an alternative strategy where the choice between the stochastic search and the gradient-based algorithm is simply based on a deterministic switch governed by the current signal estimation $^{2}$. In this framework, a particular attention is given to the effects of the choice of the parameters $\sigma_{0}$ and $\gamma$ in the activation function $\alpha$ defined in eq. (12) and the importance of the term depending on the angular difference with the previous gradient estimation. To study these effects we consider a different signal, with a slower decay and a higher measurement noise $(\xi=1)$. In this way, besides the pure exploration phase in search for a signal, there is also a larger zone where the signal is of the same order of the noise, and so where the gradient is more difficult to estimate correctly. More formally, the signal is modeled as a Cauchy-like distribution:

$$
\sigma(\mathbf{r})=a \frac{b^{2}}{x^{2}+y^{2}+b^{2}}
$$

where the $a$ and $b$ have been fixed to 10 and 30 respectively and the source location is also in this case in $(0,0)$.

A first analysis is carried out varying the value of $\sigma_{0}$ and comparing the failure rate for a fixed mission time of the two different strategies: $i$ ) the standard GCRW algorithm and ii) the deterministic switch where the probability $\alpha_{t}$ simply becomes a binary variable as follows:

$$
\alpha_{t}(\bar{\sigma})=\left\{\begin{array}{ll}
1 & \text { if } \quad \bar{\sigma}_{t} \geq \sigma_{0} \\
0 & \text { if } \quad \bar{\sigma}_{t}<\sigma_{0}
\end{array} .\right.
$$

For the GCRW algorithm, three instances corresponding to three different values of $\gamma$ have been taken into account, namely $\gamma=3,5$ and 10 . The results are provided in Fig. 7, where 500 trials, with an initial distance of the formation from the source of $200 \mathrm{~m}$, have been analyzed. A first important consideration is that the failure rate for the proposed GCRW algorithm is zero from any value of $\sigma_{0}$ greater than 3 , while basically 1 for $\sigma_{0}<2$. This can be interpreted as follows: for values lower than 2 , the system trusts the gradient

\footnotetext{
2 We would like to remark that direct comparisons with the more standard source-seeking algorithms presented in Section 2 are not feasible since they rely either on a reliable gradient estimation or on a flow transporting the signal, neither of them available in the studied scenario.
} 


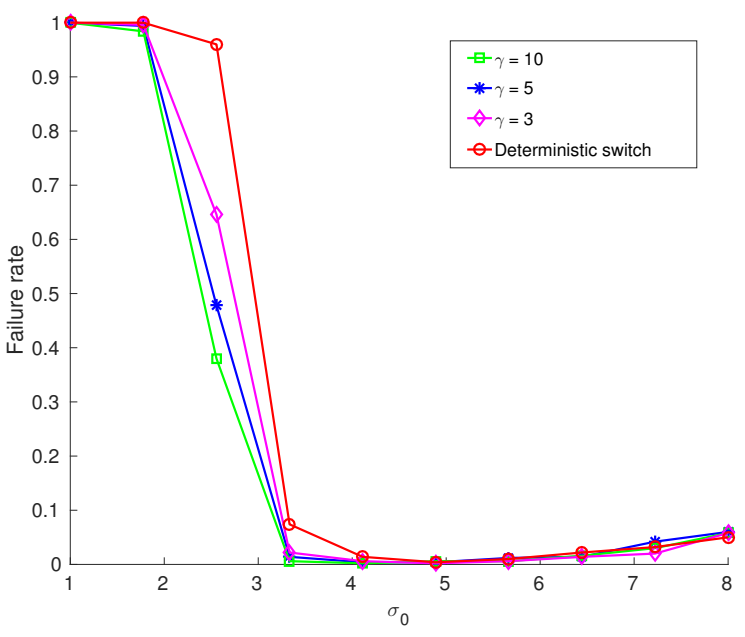

Fig. 7 Failure rate for a mission time of $10^{3} s$ as a function of $\sigma_{0}$. In the GCRW algorithm $\alpha_{t}$ is defined as in eq. (12), while for the deterministic switch the expression reported in eq. (19) is used. The results correspond to 500 trials.

estimation even in regions where it is absolutely unreliable (signal too close to noise level), misguiding the formation; on the other side, larger values are safe margins to start isolating the signal from the noise level. It is also worth noting that the exploration capabilities of the team are sufficient to eventually locate the source even for very conservative choices of $\sigma_{0}$, showing the robustness of the algorithm. In many real cases, a reliable knowledge of the signal strength and/or the noise level is indeed not precisely available. As a result, a suitable choice of this parameter is not easy and a conservative value may be a safer choice. Secondly, it is already possible to see a difference between the deterministic switch and the GCRW algorithm. Especially for lower values of $\sigma_{0}$, the former is significantly less stable, trusting too much inaccurate gradient estimations, leading to higher failures rates, while the results for different $\gamma$ values do not differ much from each other.

The difference between these strategies becomes then more clear considering the average time required to locate the source. For the same scenario, we restrict our attention to the values of $\sigma_{0}$ for which failures are negligible and the mission times are averaged over the same 500 trials. Here, we can see that the probabilistic switch employed by the GCRW algorithm, including the trust term expressing the correlation between consecutive gradient estimation, makes the convergence to the source faster in the entire range of analyzed values of $\sigma_{0}$ and regardless the choice of $\gamma$ with respect to a deterministic switch. Moreover, the lowest value reached is provided by the GCRW algorithm with $\gamma=3$, i.e. corresponding to the smoothest activation function and so the smoothest transition between the two modes. As a final conclusion, we can note that, even though an optimal value is clearly present for every different 


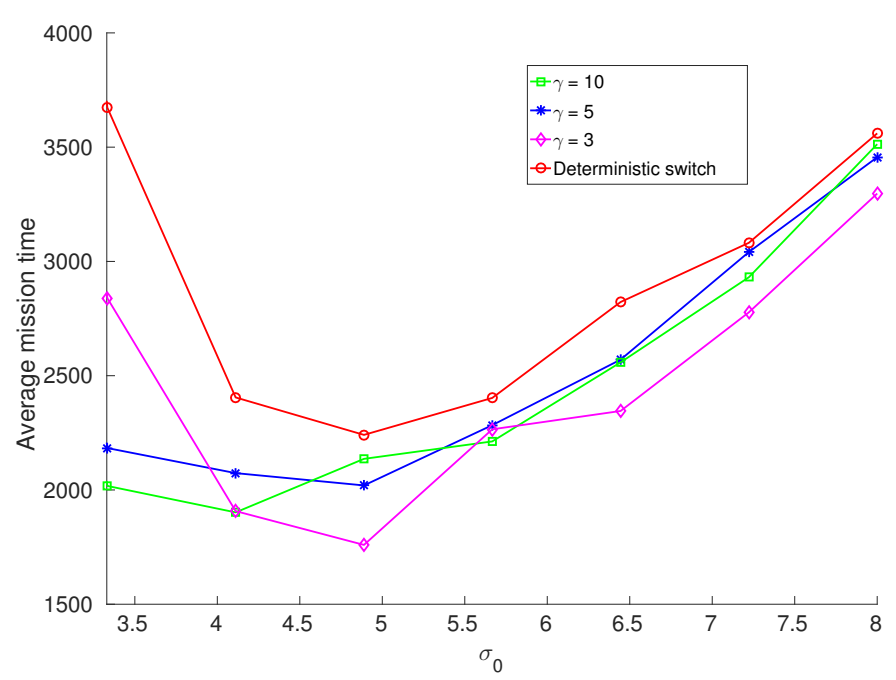

Fig. 8 Average time necessary to complete the mission as a function of $\sigma_{0}$. The reported values are averaged over 500 trials.

$\gamma$ value, the average mission time varies only slowly in terms of $\sigma_{0}$, showing again a certain robustness with respect to its choice.

Finally, we want to discuss how standard optimization algorithms might be implemented in this case and how they would perform. The important constraint that is hard to match is to drive the entire team to the source and not only a single robot or a subset of them. Without any communication constraint this could be obtained with a large spread of the robots over the environment allowing also a larger search capabilities and approaches such as the Particle Swarm Optimization (PSO) algorithm could be suitable for the problem. However, taking into account strong limitations in the communication (i.e. a maximum communication range), these solutions are not trivially adaptable anymore.

A different option is represented by global gradient-free optimization algorithms, such as Simulated Annealing based approaches. However, we consider here on-line applications where, to test possible candidate states, the robots need to physically travel to take measurements before deciding whether or not accept them. This produces very irregular and expensive paths. To show an example, we tried to adapt this method to our scenario: keeping the circular formation, at each iteration the maximum signal strength measured by the robots is taken into account for the optimization and, when accepted, the position of the corresponding robot becomes the new formation center. Then the formation follows a standard simulated annealing algorithm. Fig. 9 shows two typical solutions obtained by the optimizer in the same scenario presented in Fig. 1. Clearly, the need to test many possible states to converge to the final - global - optimum makes the system travel much more (and in a more irreg- 

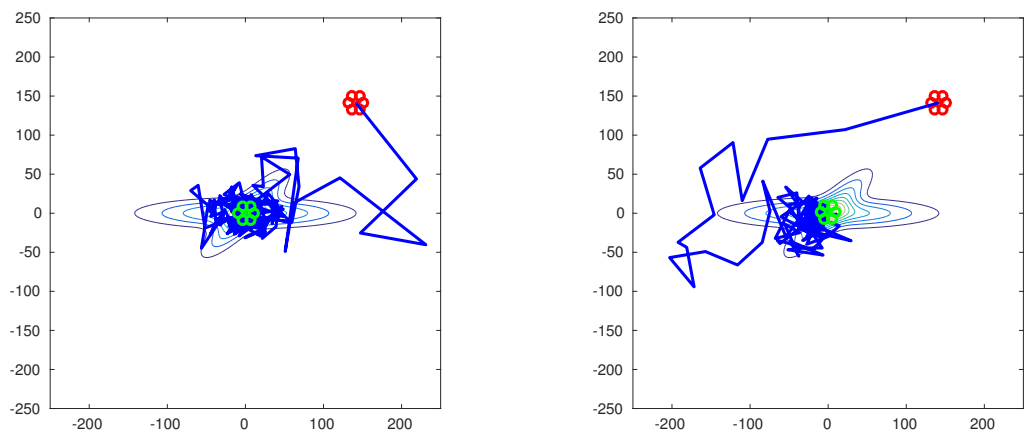

Fig. 9 Two instances of final path obtained by a simulated annealing approach to converge on the source.

ular way) than with our approach. Including the traveling time, the resulting average convergence time is in this case several times the one obtained with the GCRW algorithm.

\section{Conclusions}

A new strategy to search for a weak, noisy signal and locate its source using a team of mobile robots has been presented in this paper. The robots move in a circular formation and exchange information to cooperatively estimate the signal gradient based on noisy measurements, while respecting communication constraints. The formation center follows a correlated random walk which ensures good space filling properties to efficiently search for a zone of reliable signal strength. To deal with the exploration vs. exploitation problem, the estimated gradient plays the role of a bias in the probability distribution of the random walk direction, allowing the team to have a smooth transition between the two strategies and to increase the robustness with respect to a deterministic switch. Results in simulations showed the effectiveness of this algorithm.

In the future, we intend to better investigate possible variations in the algorithm to increase its performance, such as a varying formation radius along the mission, which would allow the team to improve either its exploration or estimation capability depending on the measured signal. An adaptive selection of $\sigma_{0}$ instead of an a priori choice is also a line worth investigating further. Finally, studying different signal models, relaxing the convexity assumption and including the capability of locating multiple sources would be relevant extensions for many real applications. 


\section{References}

1. T. Balch and R. C. Arkin. Behavior-based formation control for multirobot teams. IEEE Transactions on Robotics and Automation, 14(6):926-939, 1998.

2. F. Bartumeus, J. Catalan, G. Viswanathan, E. Raposo, and M. Da Luz. The influence of turning angles on the success of non-oriented animal searches. Journal of Theoretical Biology, 252(1):43-55, 2008.

3. B. Bayat, N. Crasta, A. Crespi, A. M. Pascoal, and A. Ijspeert. Environmental monitoring using autonomous vehicles: a survey of recent searching techniques. Current Opinion in Biotechnology, 45:76-84, 2017.

4. L. Briñón-Arranz, A. Seuret, and C. Canudas-de-Wit. Cooperative control design for time-varying formations of multi-agent systems. IEEE Transaction on Automatic Control, 59(8):2283-2288, 2014.

5. L. Briñón-Arranz, L. Schenato, and A. Seuret. Distributed source seeking via a circular formation of agents under communication constraints. IEEE Transactions on Control of Network Systems, 3(2):104-115, 2016.

6. D. Chang, W. Wu, D. R. Webster, M. J. Weissburg, and F. Zhang. A bio-inspired plume tracking algorithm for mobile sensing swarms in turbulent flow. In IEEE International Conference on Robotics and Automation (ICRA), pages 921-926, 2013.

7. M. Dunbabin and L. Marques. Robots for environmental monitoring: Significant advancements and applications. IEEE Robotics \& Automation Magazine, 19(1):24-39, 2012.

8. H. Hajieghrary, A. Hsieh, and I. B. Schwartz. Multi-agent search for source localization in a turbulent medium. Physics Letters A, 380(20):1698-1705, 2016.

9. V. Kumar, D. Rus, and S. Singh. Robot and sensor networks for first responders. IEEE Pervasive computing, 3(4):24-33, 2004.

10. W. Li, J. A. Farrell, S. Pang, and R. M. Arrieta. Moth-inspired chemical plume tracing on an autonomous underwater vehicle. IEEE Transactions on Robotics, 22(2):292-307, 2006.

11. L. Marques, U. Nunes, and A. T. de Almeida. Particle swarm-based olfactory guided search. Autonomous Robots, 20(3):277-287, 2006.

12. P. Ogren, E. Fiorelli, and N. E. Leonard. Cooperative control of mobile sensor networks: Adaptive gradient climbing in a distributed environment. IEEE Transactions on Automatic control, 49(8):1292-1302, 2004.

13. Z. Pasternak, F. Bartumeus, and F. W. Grasso. Lévy-taxis: a novel search strategy for finding odor plumes in turbulent flow-dominated environments. Journal of Physics A: Mathematical and Theoretical, 42(43), 2009.

14. C. S. Patlak. Random walk with persistence and external bias. The bulletin of mathematical biophysics, 15(3):311-338, 1953.

15. A. M. Reynolds. Bridging the gulf between correlated random walks and lévy walks: autocorrelation as a source of lévy walk movement patterns. Journal of the Royal Society Interface, 7(53):1753-1758, 2010.

16. R. A. Russell, A. Bab-Hadiashar, R. L. Shepherd, and G. G. Wallace. A comparison of reactive robot chemotaxis algorithms. Robotics and Autonomous Systems, 45(2):83-97, 2003.

17. D. K. Sutantyo, S. Kernbach, P. Levi, and V. A. Nepomnyashchikh. Multi-robot searching algorithm using lévy flight and artificial potential field. In IEEE International Workshop on Safety Security and Rescue Robotics (SSRR), pages 1-6, 2010.

18. M. Vergassola, E. Villermaux, and B. I. Shraiman. Infotaxis as a strategy for searching without gradients. Nature, 445(7126):406, 2007.

19. G. Viswanathan, V. Afanasyev, S. V. Buldyrev, S. Havlin, M. Da Luz, E. Raposo, and H. E. Stanley. Lévy flights in random searches. Physica A: Statistical Mechanics and its Applications, 282(1):1-12, 2000.

20. G. Viswanathan, E. Raposo, F. Bartumeus, J. Catalan, and M. Da Luz. Necessary criterion for distinguishing true superdiffusion from correlated random walk processes. Physical Review E, 72(1), 2005.

21. G. M. Viswanathan, S. V. Buldyrev, S. Havlin, M. Da Luz, et al. Optimizing the success of random searches. Nature, 401(6756):911, 1999. 